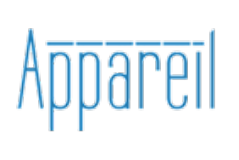

Appareil

13 | 2014

Trauma et création

\title{
Blanches et Plis, Frontières, Alphabet
}

\section{Germain Rœsz}

\section{(2) OpenEdition}

Journals

Édition électronique

URL : http://journals.openedition.org/appareil/2039

DOI : 10.4000/appareil.2039

ISSN : 2101-0714

Éditeur

MSH Paris Nord

\section{Référence électronique}

Germain Rœsz, «Blanches et Plis, Frontières, Alphabet », Appareil [En ligne], 13 | 2014, mis en ligne le 22 juillet 2014, consulté le 30 juillet 2020. URL : http://journals.openedition.org/appareil/2039 ; DOI : https://doi.org/10.4000/appareil.2039

Ce document a été généré automatiquement le 30 juillet 2020.

\section{(c) (i) () $\Theta$}

Appareil est mis à disposition selon les termes de la Licence Creative Commons Attribution - Pas d'Utilisation Commerciale - Pas de Modification 4.0 International. 


\title{
Blanches et Plis, Frontières, Alphabet
}

\author{
Germain Rœsz
}

\section{Notes sur Blanches et Plis}

1 Commencer à peindre n'est pas le commencement de la peinture. C'est tout au plus s'engager dans un espace où tout est recherche et volonté de comprendre. Cela se joue entre l'expérimentation matérielle, une sorte d'apprentissage, puis l'évaluation de ce que cela fait entre l'événement de la vie et l'expression de la peinture. Longtemps, c'est un écart considérable qu'il s'agit de réduire, lentement.

2 Les peintures blanches sont comme une peau, comme une mémoire longtemps inavouée de suaires qui furent bien réels, d'une sorte de chuchotements qui ramènent à la vie. Les plis dont je mesure encore aujourd'hui la force et la nécessité, signifient cette face cachée que je recherche, que j'essaye de faire surgir, mais c'est aussi donner à la peinture cette liberté de se faire, de se chercher, de trouver ses chemins, ce que je poursuis et concrétise aujourd'hui dans la série Alphabet. J'ai toujours plié la peinture en des formes différentes : les diptyques, les polyptiques, les pliages, voire les froissages qui donnent des temporalités décalées, montrent la peinture en train de se faire, conduisent à travailler en aveugle, à prendre des décisions de marquages de l'espace et à accepter une sorte de chaos qui s'organise à nos dépens.

3 La répétition des formes, des signes vient de la musique répétitive de P.Glass, de S. Reich, d'une volonté mathématique de travailler sur un système matriciel, d'un protocole où chaque couleur se fait à partir de la couleur existante. 
Dans la brume des effarements

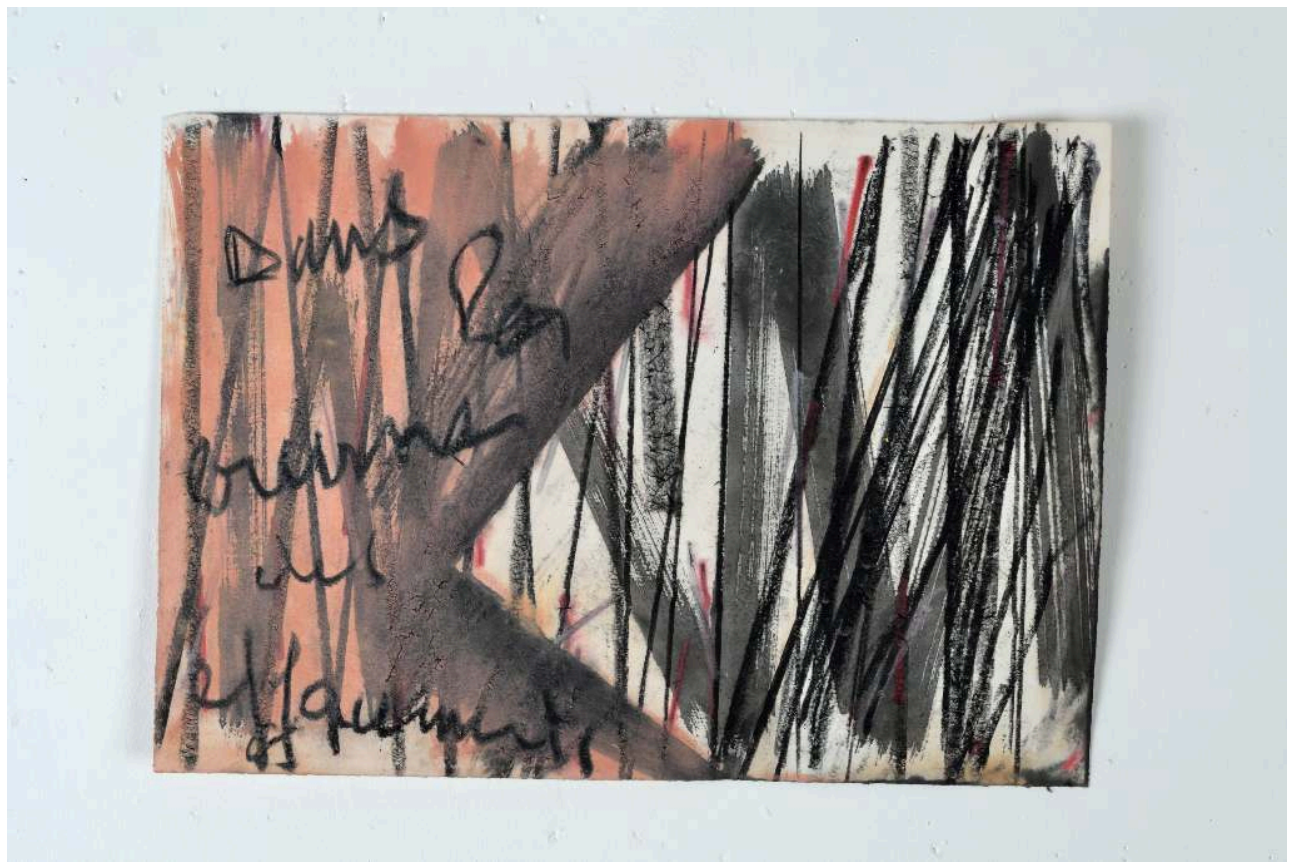

$29,2 \times 37,2 \mathrm{~cm}$, acrylique fusain sur arches

Photo Hess

Peinture réalisée dans la nature






\section{Notes sur la série Frontières}

4 Retourner aux gestes de mon travail. En faire une sorte d'inventaire, de réplique, de citation qui réinvente pourtant toujours les gestes de la peinture. Dans cette série, l'idée de l'écran est très présente. Cela se joue entre transparence et opacité, ce n'est jamais une perspective, toujours une épaisseur, je dirai visible, parfois métaphysique, mais en tous les cas toujours dans la proximité de la peinture à reconstruire et à poursuivre. On y perçoit assez clairement mes amours de la géométrie, du suprématisme, du Stijl, de Léger, d'Arp et paysagesques dont j'aime l'ironie. C'est peint sur du plexiglas en recto et en verso avec pour fond un bois peint lui aussi. C'est une peinture des échos multipliés.

\section{Notes sur la série Alphabet}

Acrylique sur bois. La couleur y est jetée, déposée, raclée, elle est liquide ou épaisse, elle trouve son chemin, fait une partie des mélanges. Elle est parfois contrariée par des ossatures géométriques, construites, volontaires mais aussi en dépendance et en accord des formes trouvées. Elle retrouve les couleurs de l'histoire de la peinture, de la caverne, du lisse, du clair et du sombre, de la forme elliptique ou allusive, d'une abstraction originelle. En aucun cas il ne faut y voir des lettres, juste des scansions, des espaces, des formes qui fondent une langue dans la langue.

\section{Fuvres intermédiaires}




\section{Équilibre précaire}

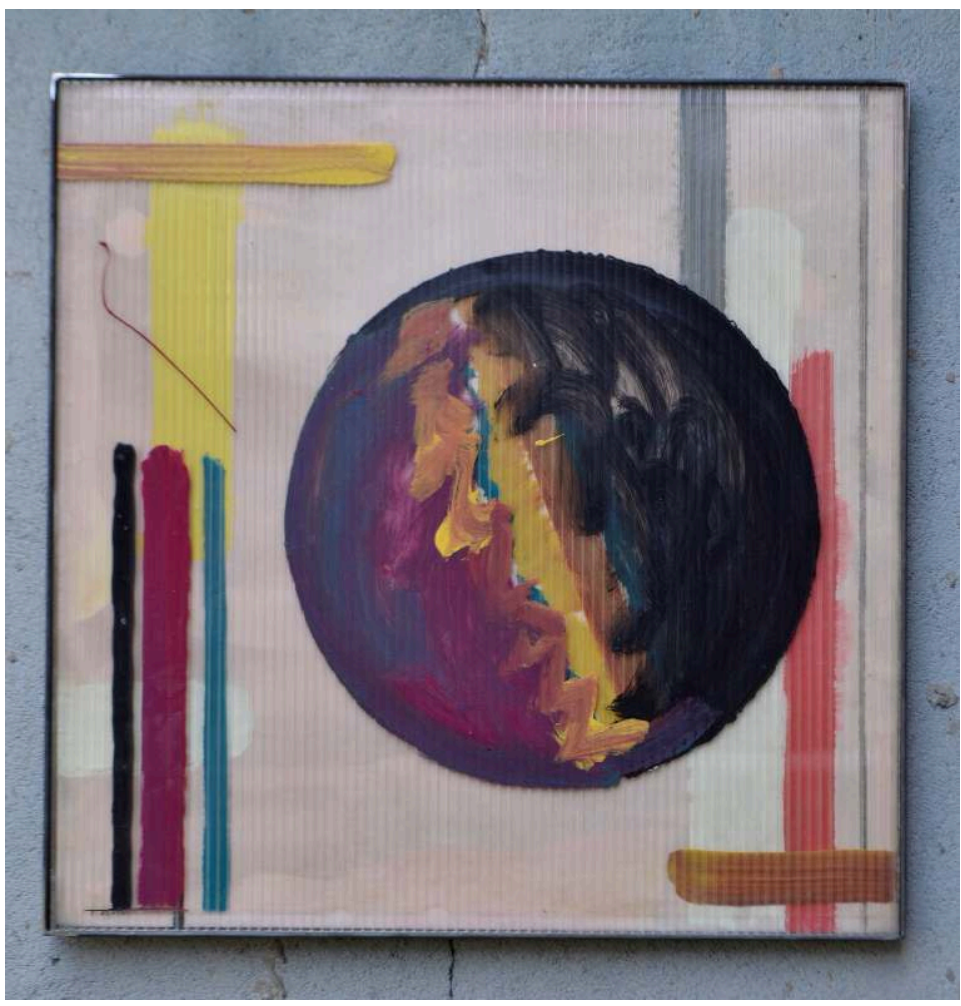

53,7 × 53,7 cm, 1998-2011

PHOTO HESS 
Tropisme

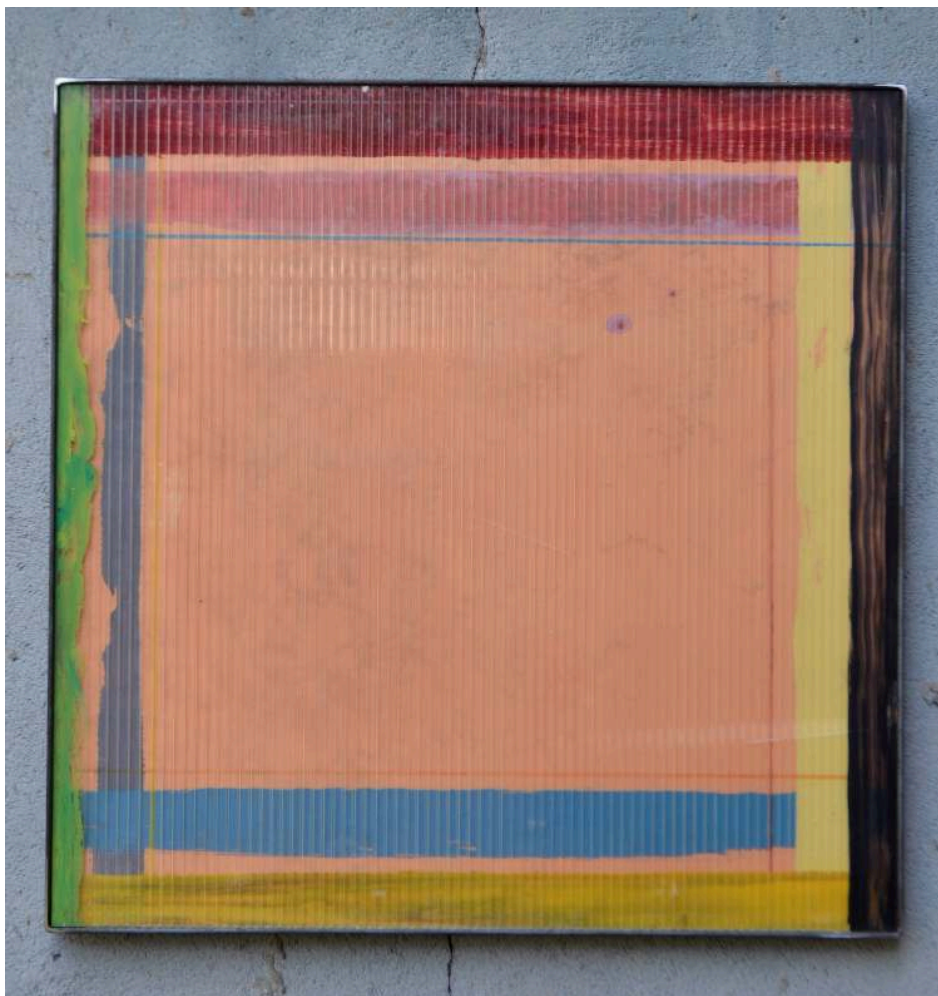

53,5 x 53,5 CM, COLL. C. BOHI, 1998-2011

Photo Hess 


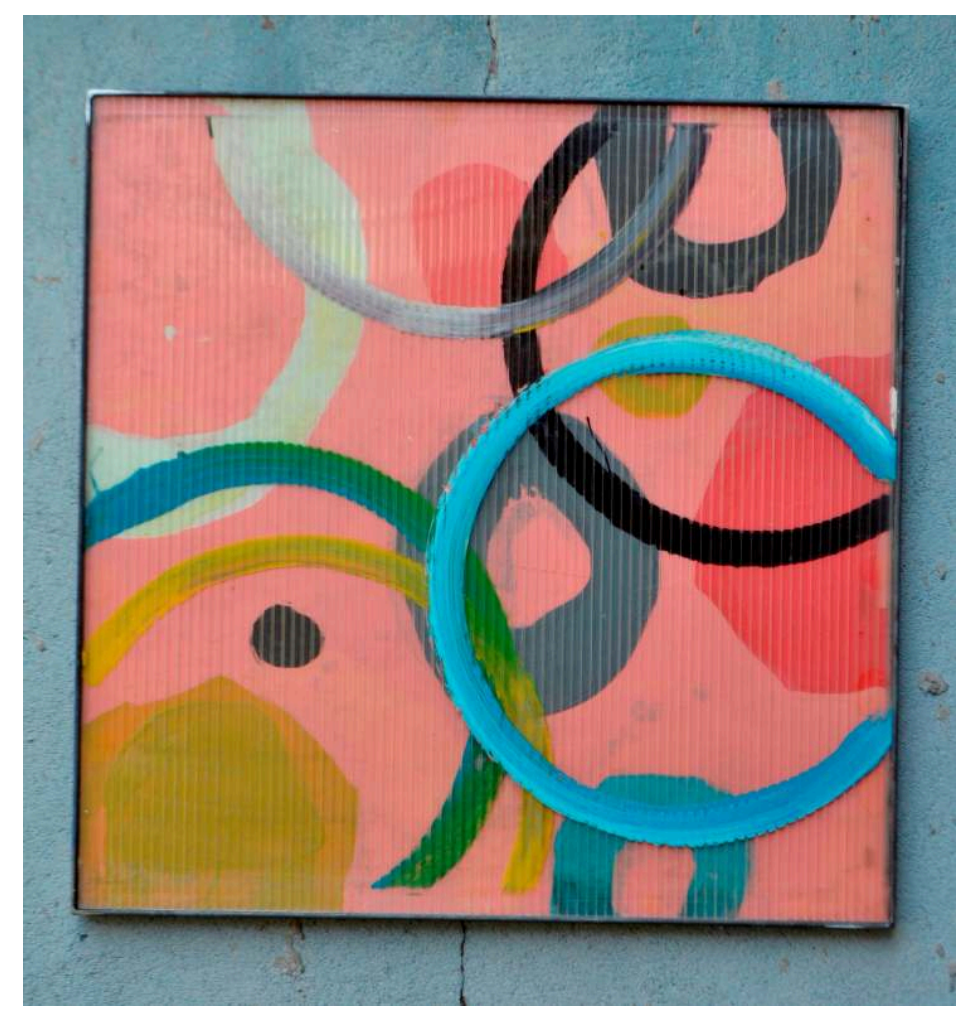

$53,5 \times 53,5 \mathrm{~cm}, 1998-2011$

Photo Hess

\section{Notes d'atelier pour Chine (extraits)}

Il s'agit de réflexions à propos d'une suite de peintures monumentales commencées début août 2010, pour plusieurs expositions en Chine. Cela concerne le pli dans mon histoire et pour aujourd'hui.

7 Sur les procédures. Plier la toile en deux. Ce pli est un horizon et un abîme. Garder l'idée de la hauteur : du soleil et de la terre. Le regard s'allège et s'ancre. Poser un cercle en bois (un de mes outils) dans la partie la plus large. Plier une frange en triangle: peindre en vert. J'ai déjà les couleurs, comme d'habitude. L'intérieur du cercle en jaune. Des gouttes violacées, blanchâtres. Du rouge à l'extérieur du cercle (un vermillon ambigu tirant vers le carmin). J'arrose l'ensemble. Un titre vient, possible : À la limite des terres je (me) parle.

8 La question du sens se pose. Il y a dans cette manière de faire du recto/verso. Choisir l'un ou (et) l'autre. Ce que font les couleurs n'est pas visible, pas totalement visible. Il y a aussi à choisir la verticalité ou l'horizontalité. Ici le désir du soleil et de la terre prime et donne la verticale. Au matin, les deux cercles (il y en a deux du fait du pliage) qui apparaissent au dépliage irradient en jaune et rouge.

9 À droite les lames-triangulées rouge/vert s'opposent en points acérés.

Je sais aussi que c'est au-delà de la terre, de la boue, c'est dans l'eau, dans l'entremêlement des formes et des couleurs. Espace.

Les toiles se font au sol. Je suis penché sur les toiles, souvent à la limite du déséquilibre. 
Je suis parfois à genoux dans la peinture, sur la peinture, pour une ligne, pour un mot, pour une phrase. Les mains et le corps enduits de couleurs.

Je cherche à faire une peinture qui bouleverse mon propre équilibre. Autre chose est l'imbrication de la couleur, des lignes, des formes et du texte poétique. Dans le temps de peinture (de l'exécution) les phrases se déroulent en continu. C'est un poème qui parle le faire, l'ailleurs du faire, le contexte et l'oubli.

11 J'attends que la couleur diffuse, se diffuse, se mêle. Qu'elle laisse la trace du passage, qu'elle dessine davantage le pli. Cette attente est une suspension, un rêve éveillé, un texte qui naît dans le lent mouvement de l'eau de la couleur.

12 Au matin les heures passées ont lié les couleurs comme un brouillard, comme l'aube qui se lève, qui se livre, grise et argentée.

13 Je retourne la toile. Elle s'est faite à l'envers, dans le dedans des procédures. Une toile c'est mince, c'est un miroir qui frappe l'ombre.

14 À la recherche d'une certaine efficacité par le pliage. Efficacité signifie rendre visible une base (construction) qui est la plus simple possible. La complexité naîtra du dépliage. Dessous, dessus, entre, devant, derrière, dans l'infra du tissu.

J'ai la conscience que c'est un drap qui enveloppe les couleurs.

On saisit un chemin qui nous perd très rapidement. Le sentiment est que dès qu'une procédure (architecture, dessin, dessein) est comprise il faut revenir en arrière pour en comprendre une autre. Dans ce retour en arrière le même chemin ne se fait jamais.

17 [...] Je me rends compte aussi que je suis dans une métaphore cosmogonique. Au fond, ma critique politique du monde $\left(\mathrm{m}^{\prime}\right)$ oblige dans ma peinture à retrouver une essence de paix, de joie, d'exaltation des couleurs. Peut-être s'agit-il d'une approche de l'origine. Chaque chemin de peinture fonde cette origine, la perçoit, l'effleure, puis la perd. C'est pour cela qu'une autre toile alors est entamée.

Cette entame dit aussi que tout est déjà commencé. Cette entame est une fissure.

19 L'invention que je peux défendre est celle qui présente une épaisseur (une peinture qui chaque fois dit des choses différentes, une peinture consciente de sa relation à l'histoire, une peinture qui revient à son auteur, où il se découvre, se pense, se modifie, se fâche).

20 La vie d'un homme et la vie des hommes couvrent (couvent) des territoires, des expériences totalement différentes, multiples. Pourquoi vouloir resserrer cela dans une procédure unique? Ce que je cherche est le déploiement et le rendu-visible de ce déploiement.

21 La toile est comme une empreinte de vie. Elle parle dans les années passées, elle se pare de l'événement, de la durée, de l'émotion, de la fragilité. Des dessins naissent par rapport à cette peinture, des dessins que je ne regarde plus après, qui me servent juste d'élan, de jet.

Dans la toile (celle qui s'intégrera à la plus grande), j'écris et je modifie mes notes.

Par le pliage (puis le dépliage) le dessin (la limite) se sépare, se distrait, se met à distance.

Nous regardons le paysage recomposé, ses frontières anciennes apparaissent incompréhensibles et insaisissables. 
25 L'eau tient un rôle important. C'est comme un étang, puis une mer - avec flux et reflux -, de la pluie légère, crachotée, ou torrentielle, de la couleur qui inonde le sol audelà de la toile.

26 À vrai dire j'aime la présence de ces traces, de ces restes sur le sol du travail. Il a la même fonction que la couleur résiduelle que je conserve (dans mes seaux) pour poursuivre mon travail de peintre. Le sol peint est la peinture déjà là dont il faut partir. 